\title{
Popliteal Vascular Injuries
}

\author{
Mohammed Al Saeed \\ Department of Surgery, College of Medicine and Medical Sciences \\ Corresponding author: Mohammed Salem Al Saeed, Telephone: 00966505370360, email: dr.alsaeed@mail.com
}

\begin{abstract}
:
Background: Injury of the popliteal vessels is one of the most limb-threatening peripheral vascular injuries. The high morbidity after popliteal vascular injury can be explained by the fact that the popliteal artery is an end artery with an insufficient collateral supply; in addition, the popliteal vein provides the bulk of lower leg and foot drainage. Delays in diagnosis and interference in addition to associated complex tissue injuries would be associated with high amputation rate. However, high level of suspicion with early recognition and management by multidisciplinary team are necessary to optimize limb salvage. The aim of this review is to discuss the different factors that may affect the morbidity, incidence, presentation and diagnostic tools, various management options, and outcome of popliteal vascular injury.
\end{abstract}

Keywords: popliteal trauma, vascular injury, outcome

\section{Introduction:}

The incidence of popliteal vascular injury is low and the vein is commonly injured with the artery ${ }^{[1-3]}$. The collateral flow around the knee is not sufficient to sustain viability after popliteal arterial injury with high amputation risk especially in blunt trauma ${ }^{[2]}$. Optimal management strategies of popliteal vascular injuries are still under investigation [3-5]. However, the advancement of imaging modalities, improved diagnostic accuracy, and the evolution of endovascular techniques offer alternative modalities to traditional surgical management ${ }^{[1-4,6-9]}$. Outcome can be improved if the lesion is early recognized and managed by multidisciplinary team ${ }^{[1,3]}$. The standards of care for patients with suspected popliteal vascular injury is urgent operative exploration in unstable patients or/and those with hard signs and arteriography is only indicated in stable patients with non occlusive lesions and in patients with soft signs only ${ }^{[1-4,9,12-14]}$. Primary repair is done if the damaged segment is less than $2 \mathrm{~cm}$ otherwise a graft is required. Veins must be repaired when possible with early fasciotomy especially in prolonged ischemia, presence of marked edema, and combined arterio-venous injury ${ }^{[1-3]}$. Dead limb must be amputated, however, amputation is considered in life threatening conditions as uncontrollable bleeding and in complex injuries ${ }^{[7]}$,The aim of this review is to discuss the different factors that may affect the morbidity, incidence, presentation and diagnostic tools, various management options, and outcome of popliteal vascular injury.

\section{Anatomical considerations:}

The popliteal artery is the continuation of the superficial femoral artery as it emerges from the adductor hiatus ${ }^{[10,11]}$. It courses posteriorly with the vein and the tibial nerve in the popliteal fossa between the medial and lateral heads of the gastrocnemius and popliteus muscles and only ceiled by subcutaneous tissue $[1-3,10]$. This relatively superficial position and the lack of protection of the artery by any overlying structures makes the blunt trauma produces injury of greater magnitude than would occur to a more protected arteries subjected to the same force $^{[2,3,10,11]}$. The popliteal vessels are tethered to the distal femoral shaft at the adductor hiatus and to the proximal tibia by the tendinous arch of the soleus muscle making the artery anchored at either end ${ }^{[1,3,11]}$. This double fixity increases susceptibility of the artery to stretching injuries following dislocations of the knee or fracture dislocations involving the upper tibia ${ }^{[1,3]}$. Such injury may damage long segments of the vessel, though thrombosis may only occur at one point leading to underestimation of the extent of the injury at operation ${ }^{[1,3,7-9]}$.

The popliteal artery rarely trifurcates and the artery commonly divides to the anterior tibial artery and the tibioperoneal trunk and the latter courses for about one inch and further divides to the peroneal and posterior tibial arteries ${ }^{[10,11]}$. The anastomosis between the sural, geniculate, and muscular branches of the popliteal artery behind the knee and the branches of the deep femoral artery proximally and tibial arteries distally are abundant ${ }^{[7-10]}$. However, this 
collateral network is inadequateand cannot maintain sufficient circulation of the leg and foot after acute obstruction of the popliteal artery that explains the high rate of limb loss following these injuries $[1,3,6-8,10,11]$. In addition, these trivial collaterals are liable for obstruction by edematous tissues, compressing hematoma, or/and thrombosis ${ }^{[1-3]}$. Damage to collaterals may result at the time of injury or later during surgical intervention ${ }^{[2-7]}$.

Another anatomical factor which may worsen the outcome of popliteal artery injury is the difficulty to expose the vesselover its full length where the commonly formed medial incision is usually not good to access the portion of the artery directly behind the knee joint ${ }^{[7-9]}$. Though the posterior approach is beneficial toexpose the artery in the mid-fossa but exposure is poor above and below this level because of the muscles of thigh and calf ${ }^{[2,3,12-16]}$.

The venae comitantes of the anterior and posterior tibial veins confluence to form the popliteal vein at the lower border of the popliteus and travels proximally in a dense sheath along with the artery intertwining it from medial side then posterior to become on its lateral side and this proximity explains the frequent injury of the vein when the artery is injured ${ }^{[10,11]}$. The vein is also prone to iatrogenic injury as it is often a bifid and delicate vessel ${ }^{\text {[2- }}$ 6].

Incidence: The incidence of popliteal vessel injuries is low and it varies in the literature reports from 0.1 to $0.2 \%$ representing less than $40 \%$ of infra-inguinal arterial injuries and about $5 \%$ of all vascular injuries ${ }^{[1,2,5-13]}$. The mean age in most of the records is less than 40 years with significant predominance of male gender (varying from $80-91 \%)^{[1-4]}$. Controversial data are reported regarding the predominant type of injury ${ }^{[1-10,12-35]}$. According to the NTDB reports, blunt trauma is the main cause of popliteal arterial injury representing more than $60 \%$ and is associated with fractures or/and dislocation where traction, avulsion, or injury of the vessels by bony fragments were recorded ${ }^{[12,16]}$. Similar results were reported by Harrell et al. ${ }^{[13]}$. The higher incidence of blunt trauma as a cause of popliteal artery injury in the previous reports may be explained by the fact that most trauma centers do not treat a large volume of penetrating trauma ${ }^{[13]}$. Frykberg et al. ${ }^{[3]}$, Fabian et $a l \cdot{ }^{[5]}$, Hafez et al..$^{[7]}$, and Asensio et al ${ }^{[14]}$, reported a higher incidence of penetrating trauma, however, the number of cases in the previous studies are significantly less than that recorded in the NTDB reports. Table 1 shows the predominance of blunt trauma in large NTDB series.

Table 1: Blunt vs. penetrating injuries in previous studies

\begin{tabular}{lccc}
\multicolumn{1}{c}{ Study } & Year & $\begin{array}{c}\text { Penetrating } \\
\text { injury }\end{array}$ & $\begin{array}{c}\text { Blunt } \\
\text { injury }\end{array}$ \\
Fabian $^{[5]}$ & 1982 & 125 & 40 \\
Snyder $^{[31]}$ & 1982 & 81 & 29 \\
Orcutt $^{[32]}$ & 1983 & 20 & 17 \\
Armstrong[ $^{[33]}$ & 1988 & 60 & 16 \\
Martin $^{[4]}$ & 1994 & 26 & 14 \\
Degiannis $^{[35]}$ & 1995 & 35 & -- \\
Harrell $^{[3]}$ & 1997 & 0 & 38 \\
Melton $^{[34]}$ & 1997 & 62 & 40 \\
Mullenix $^{[17]}$ & 2006 & 543 & 852 \\
Callcut $^{[19]}$ & 2009 & 34 & 2 \\
Kauvar $^{[12]}$ & 2011 & 220 & 431 \\
Cooper $^{[1]}$ & 2018 & 21 & 26
\end{tabular}

Corneille et $\boldsymbol{a l} .{ }^{[17]}$ reported in their study about pediatric lower extremity vascular injury that popliteal artery injury is exceptionally rare, only accounted for $19 \%$ of the vascular injuries below the knee and commonly iatrogenic.

Diagnosis: Primary survey for trauma patient with temporary vascular control can be achieved by applying pressure to the vessel proximal to the injury or/and the tourniquets, the latter may be helpful in the operating room, but should be limited to bleeding patients in the prehospital and field environments who are not responsive to direct pressure $[2,3,12,18]$. The use of tourniquets, especially those left for prolonged periods, may save a life but results in loss of an extremity ${ }^{[1-7,22]}$.

After initial life saving measures, the patient must be clinically examined with reexamination which remains the mainstay for identifying and treating popliteal vascular injuries ${ }^{[2,3,5,16]}$. Hard signs may be found as; observed pulsatile bleeding, visible expanding hematoma, signs of distal ischemia, arterial thrill on palpation, and bruit over the artery by auscultation. $[2,3,16]$ Presence of the previous signs, especially, if associated with neurologic deficit, delayed capillary refill, and signs of fractures or dislocation should increase the suspicion of vascular injury with a possible need for surgical exploration and repair $\left.{ }^{[1-9,} 16\right]$. Frykberg et $a l .^{[3]}$,Miranda et al. ${ }^{[16]}$, and Degiannis et $a l^{[35]}$, confirmed in their studies that the presence of hard signs during the initial physical 
examination is a reliable indicator for immediate exploration and any vascular imaging is unnecessary and may lead to prolonged ischemic time and delay definitive repair.Similar results were obtained by Harrell et al. ${ }^{[13]}$ and Wagner et al. ${ }^{[15]}$; however, the latter found that capillary refill was considered an unreliable measurement of distal perfusion. Whereby, Asensio et al.$^{[14]}$ found that in presence of cranial, thoracic or abdominal injuries associating blunt trauma, physical examination was not sensitive enough to detect vascular insult.

Presence of soft signs of vascular injury such as; minor bleeding, small to moderate size hematoma, temperature changes, ipsilateral decreased pulse, injury in proximity to major vessel, or/and presence of neurological deficit; should determine the need for close observation and monitoring provided that the ankle-brachial index $(\mathrm{ABI})$ is higher than $0.9^{[2,3,5,20]}$. If the $\mathrm{ABI}$ is lower than 0.9 , further noninvasive method can be utilized for assessing vascular injury as duplex ultrasound which has a sensitivity of $50-100 \%$ with a specificity and diagnostic accuracy over $90 \%{ }^{[20,22]}$. However, this procedure is operator dependent and its accuracy may be reduced by the presence of associated fractures, hematomas, swelling and presence of bulky dressings ${ }^{[1-9]}$.

Computerized tomographic angiography (CTA) has been proven to be valuable by many authors and angiography, is considered the gold standard specially if suspected multiple injuries as in gunshot or multiple wounds and it was found that preangiography volume resuscitation and sodium bicarbonate may help minimize complications ${ }^{[2,3,5,19]}$.

In summary, the standards of care for patients with suspected popliteal vascular injury is operative exploration in unstable patients and those with hard signs and arteriography is only indicated in stable patients with non occlusive lesions and patients with soft signs only (Table 2).

Table 2: Main lines of management

Urgent operative exploration

- Unstable patient

- Presence of hard signs as:

Active arterial (pulsatile) bleeding

Pulseless/ ischemia

Expanding pulsatile hematoma

Bruit or thrill

\section{General principles of treatment}

Non operative management is indicated in patients with asymptomatic non-occlusive lesions or who have only soft signs ${ }^{[1-9,13-16]}$. Those patients can be observed by a surgeon who is ready to perform surgical intervention if the examination findings change or if hard signs develop ${ }^{[1,3,5,13-16,19]}$.

Principles of operative management: Once operative management is indicated, rapid transport to operative room is required and a limited anticoagulation with single dose of heparin or antiplatelet drugs may be given preoperatively if there is no concurrent brain or spinal injury ${ }^{[2,3,34]}$. The Majority of the studies advocated prompt vascular repair before orthopedic intervention for combined vascular and skeletal injuries of the lower extremity in general and in popliteal vascular injury in particular ${ }^{[1-9,12,15,16,18]}$. Both legs have to be
Observation \&Arteriography

- Non occlusive lesion in stable patient

- Stable patients with soft signs only as:

Minor bleeding

Injury in proximity to major vessel

Small to moderate size hematoma

Associated nerve injury

prepared for possibility of harvesting a saphenous vein graft ${ }^{[1-9]}$. A medial approach is preferred and posterior edge of the femur is considered as the key anatomical landmark for managing popliteal injuries ${ }^{[2,3,4]}$. To expose the popliteal artery the incision is done along the anterior border of sartorius and extended inferiorly one $\mathrm{cm}$ posterior to the distal femur and proximal tibia ${ }^{[1-5]}$. Any divided muscles/tendons during exposure must be reconstructed at the end of operation to minimize postoperative disability ${ }^{[4]}$. The long saphenous vein must be preserved for its importance in the venous outflow of the lower extremity and the artery must be dissected carefully to avoid injury of the popliteal vein and saphenous nerve ${ }^{[7-9]}$. Callcut et $\boldsymbol{a l}^{\left[{ }^{[19]}\right.}$ found that intraoperative angiogram can identify limb threatening lesions, save time, and decrease amputation rate to $0 \%$ versus $30 \%$ if formal angiogram is done. After 
identification of the injury, proximal and distal control, the injured segment is resected and some surgeons prefer to perform catheter thrombectomy to obtain good inflow and backbleeding before therepair ${ }^{[5,20]}$. Sciarretta et $a l^{[2]}$ supported the findings of other studies that irrigation into the proximal and distal artery using heparinized saline would help prevent further thrombus formation. Primary anastomosis is done if the resected segment is less than two $\mathrm{cm} \cdot{ }^{[1-9,12,18,20,22]}$. In the most of cases the resected damaged segment is more than two $\mathrm{cm}$ and a tension-free anastomosis cannot be achieved anda reversed autogenous saphenous vein harvested from the contralateral leg is applied ${ }^{[1-13]}$. Frykberg ${ }^{[3]}$ documented in his report that the venous graft has a significantly lower failure rate and a better patency for anastomosis across knee joint than the prosthetic grafts in spite of being time consuming and has a donor site morbidity.The prosthetic grafts should be avoided across the knee as most of the studies found that it have lower rate of patency than veins in this particular situation and if necessary, they recommend ringed poly tetra fluoro ethylene grafts (PTFE) [1-3, 5, 6, 20]. However, Feliciano et al. ${ }^{\left[{ }^{[]}\right.}$and Jaggers et $a l^{[20]}$ found that the PTFE grafts are time saving and has the same infection rate if compared with the venous graft but have a higher failure rate reaching up to $80 \%$.Scott and Hirshberg ${ }^{[21]}$ found that exclusion and bypass in which an interposition reversed saphenous graft can be tunneled through healthy and uncontaminated tissue had the benefit of being simple, avoid massive dissection and bleeding from the injured vessels, however, its long term results are still questionable. Sciarretta et $\boldsymbol{a l} .{ }^{[2]}$ reported that extra-anatomic bypasses are rarely required and should be considered in specific circumstances such as, repairs that would be located in an infected vascular bed or in areas of extensive soft tissue injury or loss and they insisted that the injured vessel, should be removed to prevent the formation of pseudoaneurysms, which could expand, rupture or embolize. Frykberg ${ }^{[3]}$ recommended attempting primary arteriorrhaphy if the injury involves $30 \%$ of the circumference or less and care should be taken to avoid stenosis.Sciarretta et al $^{[2]}$,Feliciano et $a l .{ }^{[6]}$, and Pasch et al.$^{[22]}$ advocated liberal use of completion angiogram and they found that it can detect unsuspected problems requiring intervention in $8-12 \%$ of cases.Temporary intravascular shunt (TIVS) is a damage control procedure which may be done in complex injuriesusing Heparin-bonded shunts or self constructed shunts for rapid reperfusion [1-5, ${ }^{23]}$.TIVS gives time to stabilize fracture or/and dislocation, debride the devitalized soft tissues, harvest the saphenous vein grafts, and repair of associated venous injuries,then the shunts are removed and the injured arteries are repaired ${ }^{22}$, ${ }^{3]}$. Abou Ali et al. ${ }^{[23]}$ evaluated in their study that TIVS insertion in damage control and staged orthopedic vascular injuries allows rapid temporary reperfusion which would avoid major stress on vascular anastomosis and concluded that it had an acceptable complication burden and no associated mortality. Sciarretta et al. ${ }^{[2]}$, Feliciano et al.$^{[6]}$ and Keen et al. ${ }^{[24]}$ advocated TIVS in the presence of femur fractures that allows open reduction and internal fixation using interlocking intra-medullary nail and screws of with proper alignment of the femur and extremity lengthwise followed by removal of the shunt and popliteal artery repair. They found that this procedure prevent problems such as 'kinking' of the graft. However, the majority of authors recommended vascular repair prior to any orthopedic procedures in presence of posterior knee dislocation ${ }^{[3,22]}$.

According to Parry et al.$^{[25]}$ injured veins should be repaired in stable patient after arterial repair if technically feasible using various repairs as; lateral venorrhaphy, end to end anastomosis, or even complex repair utilizing saphenous venous graft or PTFE). Venous repair may be done before arterial repair if TIVS is used ${ }^{[2,3,23]}$.

Yelon et $\boldsymbol{a l} .{ }^{[26]}$ emphasized in their study that in unstable patients with complex injuries, ligation is safe alternative to repair and although $78 \%$ of patients developed DVT there was no significant chronic venous insufficiency and only $14 \%$ developed edema; however,they recommended long term anticoagulation.

Endovascular repair has been widely employed in the treatment of arterial injuries and it was found superior to the traditional surgical approach in being; rapid, has early recovery time, low in-hospital mortality, and with low risk of injuring important adjacent structures ${ }^{[27-29]}$. However, the use of this approach in the treatment of popliteal artery lesions remains controversial as there is little experience and further studies are required to evaluate its longterm efficacy ${ }^{[1-3]}$.

In addition, the stent in the popliteal artery though it is not contraindicated is more 
vulnerable to mechanical forces, resulting in stent fracture ${ }^{[2]}$.

Early fasciotomy would decrease the risk of compartmental syndrome and according to Keen et al. ${ }^{[24]}$; performing four compartments fasciotomy depends on the clinical situations though it may be recommended liberally by some authors. Frykberg ${ }^{[3]}$ summarized the main indications of early fasciotomy in the following conditions; presence of limb swelling, severe distal musculoskeletal injury, prolonged shock, limb ischemia more than 4-6 hours, combined arterial-venous injuries or venous ligation, and thrombosed repairs.
The decision of early amputation requires careful judgment; however, if there are; prolonged ischemic time, extensive soft-tissue injuries, associated skeletal trauma, knee dislocations, and associated nerve injury; amputation is most likely to be indicated ${ }^{[7-9,14,}$ 30]. The Mangled Extremity Severity Score (MESS, Table 3) has been used to triage the patients after sustaining extremity injuries ${ }^{[2,3,7-}$ 9, 12, 18]. Pasch et al. ${ }^{[22]}$ emphasized that reperfusion of dead limb must be avoided and primary amputation should be considered if the patient score is 7 or more.

\section{Skeletal / soft-tissue injury}

Low energy (stab; simple fracture; pistol gunshot wound) 1

$\begin{array}{ll}\text { Medium energy (open or multiple fractures, dislocation) } & 2\end{array}$

High energy (high speed missile or rifle gunshot) 3

Very high energy (high speed trauma \& gross contamination) 4

Limb ischemia (score is doubled if ischemia $>6$ hours)

No ischemia

Pulse reduced or absent but perfusion normal

Pulseless; paresthesias, diminished capillary refill

Cool, paralyzed, insensate, numb

\section{Shock}

Systolic BP always $>90 \mathrm{~mm} \mathrm{Hg}$

Hypotensive transiently

Persistent hypotension

(1)

Age

$$
<30 \text { years }
$$

$30-50$ years

$>50$ years

MESS 6 or less consistent with salvageable limb

Table 3: Mangled Extremity Severity Score (MESS)

Figure 1: Comparison of the amputation rates following blunt and penetrating injuries 


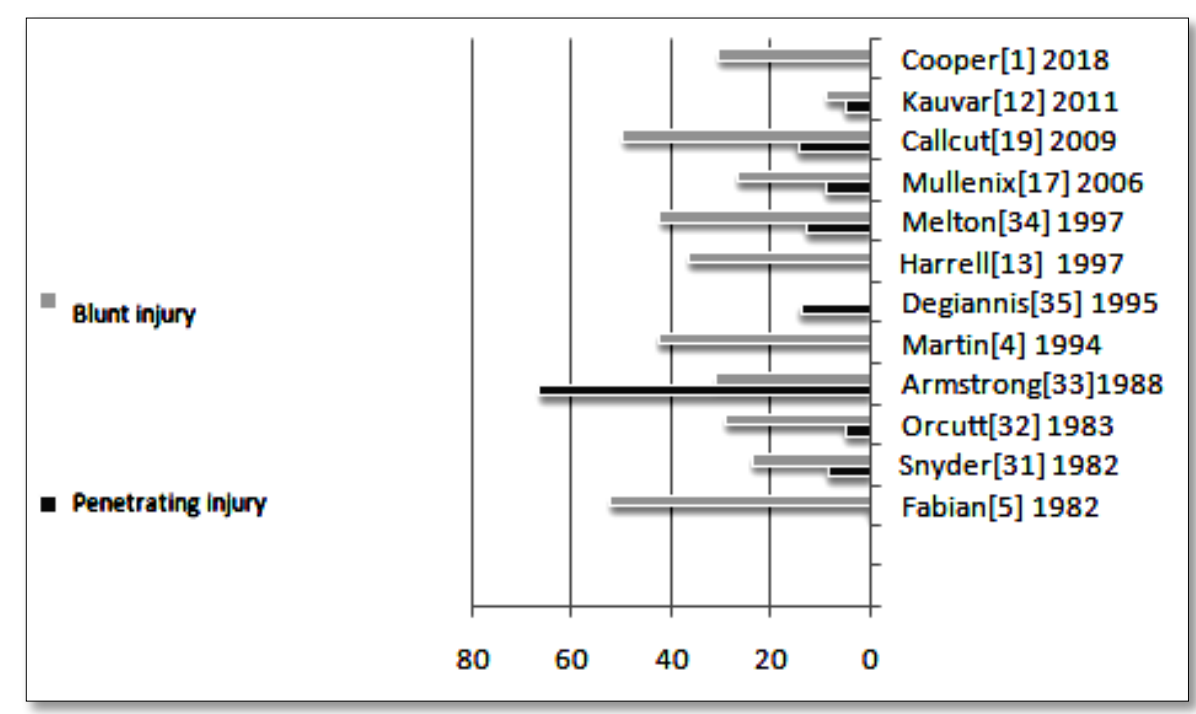

The main contributing factors to limb loss included nerve injury, prolonged ischemic times, extensive soft-tissue injuries, compound fractures, and reached $50 \%$ in crush injuries $[1,3$, ${ }^{7,8]}$. Various studies ${ }^{[1,4,5,12,13,17,19,31-35]}$ verified that blunt trauma has a significantly worse outcome if compared with penetrating trauma with higher amputation rate (Figure 1). Penetrating injuries are commonly associated with complex arterial, venous, and nerve injuries $[1-7,16]$. Several studies reported an incidence of associated venous injury in $25-34 \%{ }^{[1,2,3,5,12,18]}$. Even though penetrating injuries have shorter intensive care unit and hospital length of stay, in addition, it has a higher rate of limb salvage $[1,3$, $5,7-9,22]$. With penetrating trauma, limbthreatening vascular injuries is commonly associated with high-velocity missile injuries and the least incidence of amputation was reported following stab wound injury ${ }^{[14,15]}$.

However, marked improvement in limb salvage rate was recorded in the modern reports coming from military series; however, better outcomes and higher limb salvage rates have been reported from the civilian centers reflecting the lowervelocity mechanisms and the improved time and resources available in these centers ${ }^{[2,3,9]}$.

Lin et $\boldsymbol{a l} .{ }^{[30]}$ reported that; even with successful limb salvage a significant percentage of patients with popliteal artery injuries demonstrate neurological disabilities such as temporary or permanent peroneal nerve dysfunction with foot drop at discharge. Cooper et $\boldsymbol{a l l}^{\left[{ }^{[1]} \mathrm{emphasized}\right.}$ that early mobilization and coordinated efforts by occupational and physical therapy provide improved recovery with muscle conditioning and strengthening.

Overall mortality rate for popliteal vascular injuries ranges from $1 \%$ to $8.5 \%$ with no significant difference between blunt and penetrating groups ${ }^{[1-3,12,14,18,]}$.

To conclude; High level of suspicion with early recognition and management by multidisciplinary team are necessary to optimize limb salvage. Delays in diagnosis and interference in addition to associated complex tissue injuries would be associated with high amputation rate.

\section{References}

1. Cooper N, Roshdy M, Sciarretta J D, Kaufmann C, Duncan S, Davis J (2018): Multidisciplinary team approach in the management of popliteal artery injury. Journal of multidisciplinary healthcare, 11:399-403.

2. Sciarretta JD, Macedo FI, Otero CA et al. (2015): Management of traumatic popliteal vascular injuries in level I trauma center: a 6year experience. Int J Surg., 18: 136-41.

3. Frykberg ER (2002): Popliteal vascular injuries. Surg Clin North Am., 82:6789.

4. Martin LC, McKenney MG, Sosa JL, Ginzburg E, Puente I, Sleeman D et al. (1994): Management of lower extremity arterial trauma. J Trauma., 37:591-8.

5. Fabian TC, Turkleson ML, Connelly TL, Stone HH (1982): Injury to the popliteal artery. Am J Surg., 143:225-8.

6. Feliciano DV (2017): For the patient: evolution in the management of vascular trauma. J Trauma Acute Care Surg., 83:120512.

7. Hafez HM, Woolgar J, Robbs JV (2001): Lower extremity arterial injury: results of 550 cases and review of risk factors associated with limb loss. J Vasc Surg., 33:1212-9. 
8. Johansen $K$, Daines $M$, Howey $T$, Helfet D, Hansen S (1990): Objective criteria accurately predict amputation following lower extremity trauma. J Trauma, 30:568-72.

9. Fainzilber G, Roy-Shapira A, Wall M, Mattox K (1995): Predictors of amputation for popliteal artery injuries. Am J Surg., 170:56871.

10. Cross L, Hall J, Howdieshell, Colborn G, Gale T (2000): Clinical anatomy of the popliteal vessels. Clin Anat., 13:347-53.

11. Kropman RHJ, Kiela G, Moll FL, de Vries JP (2011): Variations in anatomy of the popliteal artery and its side branches. Vasc Endovascular Surg., 45:536-40.

12. Kauvar D, Sarfati M, Kraiss $L$ (2011) : National trauma databank analysis of mortality and limb loss in isolated lower extremity vascular trauma. J Vasc Surg., 53:1598-603.

13. Harrell DJ, Spain DA, Bergamini TM et al. (1997): Blunt popliteal artery trauma: a challenging injury. Am Surg., 63:228-32.

14. Asensio JA, Kuncir EJ, Garcia-Nunez LM, Petrone P (2006) : Femoral vessel injuries: analysis of factors predictive of outcomes. J Am Coll Surg., 203:512-20.

15. Wagner W, YellinA, Weaver F, Stain S, Siegel A (1994): Acute treatment of penetrating popliteal artery trauma: the importance of soft tissue injury. Ann Vasc Surg., 8:557-65.

16. Miranda FE, Dennis JW, Veldenz HC (2002):Confirmation of the safety and accuracy of physical examination in the evaluation of knee dislocation for injury of the popliteal artery; a prospective study. J Trauma, 52:247-51.

17. Corneille M, Gallup T, Villa C, Richa J, Wolf S, Myers J, Dent D, Stewart R (2011): Pediatric vascular injuries: acute management and early outcomes. J Trauma, 70:823-8.

18. Mullenix P, Steele S, Anderson C, Starnes BW, Salim A, Martin MJ (2006): Limb salvage and outcomes among patients with traumatic popliteal vascular injury: an analysis of the National Trauma Data Bank. J Vasc Surg., 44:94-100.

19. Callcut RA, Acher CW, Hoch J, Tefera G, Turnipseed W, Mell MW (2009): Impact of intraoperative arteriography on limb salvage for the traumatic popliteal artery injury. J Trauma, 67:252-7.
20. Jaggers R, Feliciano D, Mattox K, Graham J, DeBakey M (1982): Injury to popliteal vessels. Arch Surg., 117:657-61.

21. Scott BG, Hirshberg A (2004):

Exclusion and bypass--a new approach to popliteal artery injuries. J Trauma, 57:913-4.

22. Pasch AR, Bishara RA, Lim LT et al. (1986): Optimal limb salvage in penetrating civilian vascular trauma. J Vasc Surg., 3:189-95.

23. Abou Ali AN, Salem KM, Alarcon, LH, Bauza G, Pikoulis E, Chaer $\mathbf{R}$ A, Avgerinos ED (2017): Vascular Shunts in Civilian Trauma. Frontiers in surgery, 4, 39.

24. Keen RR, Meyer JP, Durham JR, Eldrup-Jorgensen J, Flanigan P, Schwarcz TH (1991): Autogenous vein graft of injured extremity arteries: early and late results with 134 consecutive patients. J Vasc Surg., 13:664-8.

25. Parry NG, Feliciano DV, Burke RM, Cava RA, Nicholas JM, Dente CJ et al. (2003): Management and short-term patency of lower extremity venous injuries with various repairs. Am J Surg., 186:631-5.

26. Yelon JA, Scalea TM (1992): Venous Injuries of the Lower Extremities and Pelvis: Repair Versus Ligation. J Trauma, 33:532-8

27. D'Amours SK, Rstogi P, Ball CG (2013): Utility of simultaneous interventional radiology and operative surgery in a dedicated suite for seriously injured patients. Curr Opin Crit Care, 19:587-93.

28. Worni M, Scarborough JE, Gandhi M, Pietrobon R, Shortell CK (2013): Use of endovascular therapy for peripheral arterial lesions: an analysis of the National Trauma Data Bank from 2007 to 2009. Ann Vasc Surg., 27:299e305.

29. Macedo FI, Sciarretta JD, Salsamendi J, Karmacharya J, Romano A, Namias $\mathbf{N}$ (2015): Repair of an acute blunt popliteal artery trauma via endovascular approach. Ann Vasc Surg., 29:366.e5-366.e10.

30. Lin CH, Wei FC, Levin LS, Su JI, Yeh WL (1997): The functional outcome of lowerextremity fractures with vascular injury. J Trauma, 43:480-5.

31. Snyder WH (1982): Vascular injuries near the knee: An updated series and overview of the problem. Surgery, 91:502-6.

32. Orcutt MB, Levine BA, Root HD, Sirinek KR (1983): The continuing challenge of popliteal vascular injuries. Am J Surg., 146: 758-61. 
33. Armstrong $\mathbf{K}$, Sfeir R, Rice J, Kerstein M (1988): Popliteal vascular injuries and war: are Beirut and New Orleans similar?. J Trauma, 28:836-9.
34. Melton S, Croce M, Patton J, Pritchard FE, Minard G, Kudsk K (1997): Popliteal artery trauma. Systemic anticoagulation andintraoperative thrombolysis improves limb salvage. Ann Surg., 225:518-27. 35. Degiannis E, Levy RD, Sofianos C, Florizoone MG, Saadia R (1995): Arterial gunshot injuries of the extremity: a South African experience. J Trauma, 39:570-5. 